Cómo construir la tasa de interés «arm's length» en ausencia de operaciones comparables

Building the arm's length interest rate in the absence of comparable transactions

\title{
Luis Enrique
}

Landa Fournais

Universidad Anáhuac,

México

Recibido: 6 de noviembre de 2021.

Aprobado: 31 de marzo de 2021. 


\section{Resumen}

El principio conocido como "arm's length» es la norma internacional para determinar los precios de transferencia con fines fiscales, consensuado por los países miembros de la OCDE. Uno de los temas centrales en los procesos de auditoría en esta materia es el de comprobar si las tasas de interés pactadas en operaciones de préstamo entre partes relacionadas se adhieren a este principio. Para esto, la Ley del Impuesto Sobre la Renta en México (LISR, 2013/2020) requiere evidencia de un contrato de préstamo pactado entre partes independientes en condiciones similares, con una tasa de interés que podría ser comparable. Este requisito demuestra ser oneroso puesto que la mayoría de estos contratos son de naturaleza privada, por lo que no es posible incorporarlos a la muestra de comparables. Ante la falta de un contrato entre partes independientes, este artículo presenta una opción para construir una tasa de interés basada en el principio arm's length a partir de un modelo económico, resultado de un balance entre lo establecido en la LISR (2013/2020) y las recomendaciones propuestas en el reporte Transfer Pricing Guidance on Financial Transactions (OCDE, 2020).

Palabras clave: México; precios de transferencia; impuestos; empresas multinacionales; auditorías. Clasificación JEL: M48, H2.

\section{Abstract}

The "arm's length principle» is the international standard for determining transfer prices for tax purposes agreed to by the OECD member countries. One of the main issues in the auditing processes regarding this matter is to determine whether the interest rates agreed in loan transactions between related parties comply with this principle. For this, the Mexican Income Tax Law (LISR, 2013/2020) requires the taxpayer to present evidence of a loan contract between independent parties with an interest rate that could be comparable. This requirement proves to be onerous for compliance purposes since most of these contracts are private in nature, precluding them as part of the sample of comparable transactions. In the absence of a contract between independent parties, this article presents an option to build an arm's length interest rate from an economic model, which results from a balance between compliance with the LISR (2013/2020) combined with the proposed recommendations in the Transfer Pricing Guidance on Financial Transactions report (OECD, 2020).

Keywords: Mexico; transfer prices; multinationals; taxes; audits.

JEL Classification: M48, H2. 


\section{Introducción}

El principio de plena competencia o principio de «arm's length» es la norma internacional para determinar los precios de transferencia con fines fiscales consensuado por los países miembros de la Organización para la Cooperación y el Desarrollo Económicos (OCDE). Este se encuentra establecido en el artículo 9 del Modelo de Convenio Tributario sobre la Renta y sobre el Patrimonio de la OCDE (2017a). Dado que los precios pactados en operaciones entre partes relacionadas no siempre obedecen al libre juego de las fuerzas de mercado, estos deben compararse con operaciones concertadas entre partes no relacionadas bajo condiciones similares. Una operación entre partes relacionadas cumple con el principio de arm's length si los resultados son consistentes con los obtenidos en una operación comparable entre partes no relacionadas.

Para el caso de operaciones de préstamo, las tasas de interés dependen de las características específicas de la operación, tales como las condiciones económicas, el monto, el plazo, las garantías y la solvencia del deudor, etcétera, por lo que es improbable hallar un contrato con características que pudieran considerarse lo suficientemente cercanas para efectos de comparabilidad de las tasas de interés. El problema se exacerba por el hecho de que la mayoría de los contratos de préstamo son operaciones privadas, por lo que no pueden incluirse como elementos de la muestra de comparables. Esto es particularmente relevante para el contribuyente cuando existen operaciones de préstamo con intereses devengados a cargo, puesto que, en ausencia de comparables, los intereses pueden dictaminarse como no deducibles en la declaración del Impuesto Sobre la Renta (ISR).

Para sobreponerse a este problema y como alternativa a la presentación de contratos de préstamo pactados entre partes independientes, este artículo propone una metodología para construir una tasa de interés arm's length a partir de un modelo económico conforme a las mejores prácticas presentadas en el informe Transfer Pricing Guidance on Financial Transactions (Directrices de transferencia de precios en transacciones financieras), de la OCDE (2020). Para esto, se construye la tasa de interés comparable en el marco de una auditoría fiscal aplicada a un caso hipotético, en donde una empresa multinacional mexicana otorga una serie de préstamos a su empresa subsidiaria en Brasil. La empresa es ficticia y los datos y las operaciones son hipotéticos. Sin embargo, la situación fiscal en la que se enmarca se presenta con frecuencia en procesos de auditoría en materia de precios de transferencia. De esta forma, se resalta la utilidad de la construcción de tasas de interés arm's length a partir de modelos económicos en situaciones que se presentan repetidamente en auditorías reales. 


\section{Antecedentes}

Al realizar las auditorías en materia de precios de transferencia, los intereses devengados a favor o a cargo derivados de operaciones con partes relacionadas representan ingresos acumulables y gastos deducibles, que tienen una incidencia directa sobre la base gravable. Para que el contribuyente pueda demostrar que las tasas de interés pactadas en préstamos con sus partes relacionadas se apegan al principio de arm's length, es necesario cumplir con el siguiente requisito establecido en los artículos 76, primer párrafo, fracción XII y 179, primer párrafo de la LISR (2013/2020) vigente en 2020:

Los contribuyentes que celebren operaciones con partes relacionadas están obligados a determinar sus ingresos acumulables y deducciones autorizadas, considerando para esas operaciones los precios y montos de contraprestaciones que hubieran utilizado con o entre partes independientes en operaciones comparables.

Para demostrar que las tasas de referencia provenientes de mercados financieros internacionales y nacionales se utilizan con o entre partes independientes, es necesario documentar que una entidad independiente pactó una operación de financiamiento con alguna de esas tasas para presentar la evidencia de una operación potencialmente comparable.

En un contexto de precios de transferencia, el argumento que suele presentar el contribuyente de que las operaciones que generaron intereses a favor o a cargo se pactaron a tasas que por definición son de mercado, como podrían ser, por ejemplo, CETES, TIIE o LIBOR, no es admisible para ninguna autoridad fiscal. Hacer referencia a estas tasas de interés como tasas comparables pudiera ser aceptable bajo un enfoque estrictamente financiero. Sin embargo, en materia de precios de transferencia, los artículos anteriormente citados de la LISR (2013/2020) exigen «un paso adicional» y establecen que es necesario demostrar que las tasas se definieron entre partes independientes para pactar operaciones en condiciones similares. Para esto, es necesario que el contribuyente presente evidencia de un contrato de dominio público entre partes independientes, en donde se formalice una operación con referencia a la tasa relevante de mercado. El problema para satisfacer este requisito es que la mayoría de los contratos entre partes independientes son acuerdos confidenciales entre empresas privadas. Por eso, quedan excluidos de la muestra de comparables, haciendo que la «oferta residual» de dominio público sea escasa y de poca utilidad. Mientras no existan operaciones 
potencialmente comparables, no se podrá cumplir con lo establecido en la LISR (2013/2020).

Adicionalmente al requisito de presentar contratos celebrados entre partes independientes, el contribuyente debe cumplir con lo establecido en el artículo 179, inciso I, Fracción (a), de la LISR (2013/2020):

Para los efectos de esta Ley, se entiende que las operaciones o las empresas son comparables, cuando no existan diferencias entre éstas que afecten significativamente el precio o monto de la contraprestación o el margen de utilidad a que hacen referencia los métodos establecidos en el artículo 180 de esta Ley, y cuando existan dichas diferencias, éstas se eliminen mediante ajustes razonables. Para determinar dichas diferencias, se tomarán en cuenta los elementos pertinentes que se requieran, según el método utilizado, considerando, entre otros, los siguientes elementos: I. Las características de las operaciones, incluyendo: a) en el caso de operaciones de financiamiento, elementos tales como el monto del principal, plazo, garantías, solvencia del deudor y tasa de interés [...].

Debido a que en la normatividad fiscal mexicana no existe ninguna guía de cómo realizar los ajustes a las operaciones de préstamo para mejorar la comparabilidad entre estas, cualquier iniciativa para realizar los «ajustes razonables» estaría abierta a debate entre el contribuyente y la autoridad fiscal para determinar la procedencia de los mismos.

\section{Marco conceptual}

Existe una cantidad abundante de literatura académica sobre los diferentes temas relacionados con precios de transferencia a la cual se puede hacer referencia, como por ejemplo, Padhi (2019), Eden et al. (2019), Silva (2019), Durst (2012) y en los manuales publicados por el International Bank for Reconstruction and Development (IBRD, 2016) y Naciones Unidas (2013). ${ }^{1}$ No obstante su relevancia, el presente artículo se basa en la literatura de la OCDE en materia de precios de transferencia,

\footnotetext{
1 La literatura en materia de precios de transferencia es extensa, por lo que hacer una revisión exhaustiva de la misma va más allá de los objetivos del presente artículo. No obstante, se citan algunas referencias que a criterio del autor contribuyen de manera importante al desarrollo de aplicaciones prácticas en forma de estudio de caso, como el que se presenta en este artículo.
} 
de la cual destacan Directrices de la OCDE aplicables en materia de precios de transferencia a empresas multinacionales y administraciones tributarias (OCDE, 2017b) a la que se hace referencia explícita en el último párrafo del artículo 179 de la LISR (2013/2020) en México, y las nuevas directrices en materia de operaciones financieras, Transfer Pricing Guidance on Financial Transactions (OCDE, 2020), que se estima que en 2022 será incorporado como un capítulo adicional a una versión actualmente en preparación de las Directrices (OCDE, 2017b).

Considerando que las dificultades para el cumplimiento del principio de arm's length en las tasas de interés pactadas en préstamos entre partes relacionadas descritas en el apartado anterior no son exclusivas para México, en febrero 2020 la OCDE publicó el informe: Transfer Pricing Guidance on Financial Transactions; Inclusive Framework on BEPS; Actions 4, 8-10 (en adelante el reporte OCDE, 2020). En este reporte, se presentan algunas guías para sobreponerse al difícil reto de ubicar contratos de préstamo entre partes independientes con características similares o con diferencias que puedan ser «subsanadas» a través de ajustes razonables para demostrar comparabilidad. En la sección C.1.2 de dicho informe, se habla específicamente acerca de las diferentes alternativas para determinar si las tasas de interés pactadas entre partes relacionadas cumplen con el principio de arm's length. En concreto, menciona lo que a continuación se explica.

\section{La aplicación del método de precio comparable no controlado (CUP, por sus siglas en inglés)}

Dado el tamaño, la extensión y profundidad de los mercados globales de fondos disponibles para préstamo, combinado con la extensa disponibilidad de información de operaciones entre partes independientes, el método CUP es la primera alternativa para determinar la comparabilidad entre tasas de interés.

Las tasas de interés que cumplen con el estándar de arm's length pueden basarse en tasas de rendimiento de transacciones alternativas en condiciones económicas similares. Por ejemplo, las tasas de interés de emisiones de bonos, que son préstamos entre partes independientes, depósitos, obligaciones convertibles, pagarés, papel comercial, etcétera. En la evaluación de estas operaciones independientes como posibles comparables, se pueden requerir ajustes de comparabilidad para eliminar o amortiguar las diferencias entre la operación sujeta a revisión y la alternativa seleccionada como comparable. Estas diferencias pueden estar relacionadas 
con las condiciones económicas, plazos, garantías o la moneda con la cual se realiza la operación.

La contribución de esta sección del reporte OCDE, 2020 es que menciona explícitamente las diferentes transacciones que existen en los mercados financieros internacionales, contra las cuales las operaciones de préstamo entre partes relacionadas pueden compararse. Particularmente, las emisiones de papel se consideran como préstamos entre partes independientes que fortalecen su candidatura a ser adoptadas como operaciones comparables. Sin embargo, el informe advierte con toda claridad sobre la necesidad de realizar ajustes para mejorar la comparabilidad entre las operaciones.

\section{El enfoque de costo de fondos}

En ausencia de información disponible sobre transacciones financieras comparables realizadas entre partes independientes, el enfoque de costo de fondos puede utilizarse como segunda alternativa para asignarle una tasa de interés arm's length a los préstamos entre partes relacionadas. Al costo de fondeo incurrido por el acreedor, se le sumaría una prima que refleje los costos de administración, una prima de riesgo y un margen de ganancia.

El reporte OCDE, 2020 hace énfasis en que un factor que debe tomarse en cuenta al aplicar este enfoque de costo de fondos es que deben considerarse ambos, el costo de fondeo del acreedor que forma parte del grupo corporativo y el costo de fondeo de otros prestamistas independientes operando en el mercado. Es decir, el costo de fondos del acreedor no es suficiente si no se compara contra el costo de fondos de otras entidades participantes en el mercado ya que, en un entorno competitivo, la entidad que solicita el acceso a los fondos podría acceder a estos bajo condiciones más favorables, por intermedio de entidades independientes externas.

\section{La aplicación de modelos económicos}

Como tercera alternativa, algunas industrias utilizan modelos económicos para calcular la tasa de interés para préstamos vinculados como una aproximación a la tasa de interés que pactarían entidades independientes en situaciones similares.

En términos generales, los modelos económicos calculan la tasa de interés como una combinación de una tasa libre de riesgo y una serie de sobretasas asociadas con 
diferentes características del préstamo, como el plazo; las comisiones, la solvencia del deudor; el margen de ganancia y, en ocasiones, los gastos de administración asociados con el préstamo.

Es importante recalcar que los resultados no representan transacciones reales entre partes independientes. Sin embargo, en situaciones en donde no se puedan identificar comparables independientes confiables, los modelos económicos pueden representar herramientas que pueden ser aplicadas de manera útil para identificar y determinar una tasa de interés arm's length para operaciones entre empresas relacionadas.

\section{Encuestas directas a instituciones de crédito}

Finalmente, el reporte OCDE, 2020 presenta la opción de buscar evidencia sobre las tasas de interés arm's length para préstamos vinculados a través de opiniones escritas de bancos independientes, en el que se dejaría constancia sobre la tasa de interés que aplicaría la banca si fuese a otorgar un préstamo similar a la empresa que se encuentra bajo examen.

Sin embargo, el reporte resalta que la evidencia proporcionada por escrito por los bancos no representa una oferta formal ni un compromiso para el otorgamiento de crédito a la empresa bajo examen. Antes de otorgar algún préstamo, la institución crediticia debe llevar a cabo el «due dilligence» (diligencia debida) y recorrer el camino interno de aprobación del crédito; ambos tienen incidencia directa sobre el costo del crédito. Debido a esto, la evidencia por escrito con esta información no debe considerarse como evidencia de los términos y condiciones arm's length que permita su comparación con las tasas de interés pactadas por el contribuyente.

\section{Consideraciones}

Con las opciones presentadas en el reporte OCDE, 2020, se abre una ventana de oportunidad para el contribuyente y para la autoridad fiscal de explorar diferentes formas de determinar la tasa de interés arm's length, como alternativa a la búsqueda de contratos entre partes independientes.

Como primer criterio para establecer comparabilidad están las tasas de interés de los diferentes productos financieros en el mercado global, siendo estos bonos, obligaciones convertibles, préstamos, pagarés, papel comercial, etcétera. Dado que en el reporte OCDE, 2020 estos productos ahora se consideran explícitamente como 
operaciones de préstamo entre partes independientes, se permite que el contribuyente y la autoridad fiscal los considere como contratos para dar cumplimiento a lo establecido en los artículos 76, primer párrafo, fracción XII y 179 primer párrafo de la LISR (2013/2020). Sin embargo, su elegibilidad para considerarse como una tasa de interés arm's length se dará cuando se realicen los ajustes necesarios para mejorar la comparabilidad, considerando las diferentes características de las transacciones seleccionadas. Es decir, la LISR (2013/2020), en el artículo 179, inciso I, Fracción (a), establece que la autoridad fiscal puede aceptar una operación como comparable, si el contribuyente demuestra la manera en el monto del principal, el plazo, las garantías y la solvencia del deudor afectan la tasa de interés de la operación y, después, procede con los ajustes correspondientes para mejorar la comparabilidad. Sin embargo, dado que no existe una guía de cómo realizar los mencionados ajustes, es improbable que el contribuyente pueda cumplir con lo establecido en el citado artículo.

El segundo criterio es el enfoque de costo de fondos, que también requiere de una investigación en cuanto a la búsqueda de transacciones comprables. Difícilmente una entidad financiera independiente revelará su costo de fondos a los clientes o a empresas que lo soliciten como dato informativo.

Dada la dificultad de aplicación de los dos criterios anteriores, la tercera opción, que consiste en aplicar modelos económicos para construir una tasa de interés que aproxime a aquella que pactarían partes independientes en operaciones similares, se convierte en una alternativa viable. Esta opción permite la construcción de tasas de interés arm's length para su posterior uso en un ejercicio de comparabilidad con las tasas pactadas por el contribuyente. La aplicación empírica de esta alternativa se presenta a continuación en el marco de una auditoría hipotética.

\section{Presentación del caso²}

Supongamos que una empresa multinacional mexicana (en adelante el contribuyente) otorga préstamos a una subsidiaria ubicada en Brasil, generando intereses a favor, los cuales son presentados por el contribuyente como ingresos acumulables

\footnotetext{
2 Los datos y las operaciones son hipotéticas. Sin embargo, el contexto fiscal suele presentarse con frecuencia en procesos de auditoría en materia de precios de transferencia. De esta forma, se resalta la utilidad de la construcción de tasas de interés arm's length a partir de modelos económicos en situaciones que se presentan repetidamente en auditorías reales.
} 
en la declaración del ISR para el ejercicio fiscal 2020. Las operaciones relevantes se presentan en la tabla 1 (ver tabla 1).

Tabla 1. Intereses devengados a favor por operaciones de préstamo en 2020

\begin{tabular}{|c|c|c|c|c|c|c|}
\hline \multirow[b]{2}{*}{ Mes } & \multicolumn{6}{|c|}{ Cálculo de los intereses devengados a favor } \\
\hline & $\begin{array}{l}\text { Monto en } \\
\text { dólares }\end{array}$ & $\begin{array}{l}\text { Tasa de } \\
\text { interés }\end{array}$ & Días & $\begin{array}{l}\text { Intereses } \\
\text { en dólares }\end{array}$ & $\begin{array}{l}\text { Tipo de } \\
\text { cambio }\end{array}$ & $\begin{array}{l}\text { Intereses } \\
\text { en pesos }\end{array}$ \\
\hline & A & B & C & $\begin{array}{c}D= \\
(A \times B / 360) \\
x C\end{array}$ & E & $\mathrm{F}=\mathrm{DxE}$ \\
\hline Enero & 5675000 & $4.20 \%$ & 2 & 1324 & 18.804 & 24900 \\
\hline Febrero & 15000000 & $4.20 \%$ & 31 & 54250 & 18.844 & 1022287 \\
\hline Marzo & 14456000 & $4.20 \%$ & 30 & 50596 & 22.318 & 1129202 \\
\hline Abril & 16543000 & $4.20 \%$ & 31 & 59831 & 24.266 & 1451847 \\
\hline Mayo & 4050000 & $4.20 \%$ & 2 & 945 & 23.423 & 22135 \\
\hline Suma & & & & 166946 & & 3650370 \\
\hline Enero & 6500000 & $4.52 \%$ & 1 & 816 & 18.804 & 15346 \\
\hline Febrero & 17650000 & $4.52 \%$ & 31 & 68698 & 18.844 & 1294540 \\
\hline Marzo & 14750000 & $4.52 \%$ & 31 & 57410 & 22.318 & 1281283 \\
\hline Abril & 16350000 & $4.52 \%$ & 30 & 61585 & 24.266 & 1494422 \\
\hline Mayo & 15400000 & $4.52 \%$ & 31 & 59940 & 23.423 & 1403980 \\
\hline Junio & 14630000 & $4.52 \%$ & 30 & 55106 & 22.299 & 1228816 \\
\hline Julio & 16100000 & $4.52 \%$ & 31 & 62665 & 22.768 & 1426752 \\
\hline Suma & & & & 366220 & & 8145138 \\
\hline TOTAL & & & & 533166 & & 11795508 \\
\hline
\end{tabular}

Fuente: documentación comprobatoria del contribuyente.

Se realizaron 12 operaciones de préstamo por montos que fluctúan en un rango de US5.675-17.650 millones, con plazos mínimos de 1 día a un máximo de 31 días. El monto total de intereses devengados en pesos fue de MX 11795 508.00.

A fin de dar cumplimiento a lo dispuesto por los artículos 76, primer párrafo, fracciones IX y XII; 179, tercero, cuarto y último párrafos y 180, primer párrafo, fracción I 
de la LISR (2013/2020) vigente en 2020 en materia de precios de transferencia, el contribuyente buscó tasas de referencia en dólares en el mercado de Estados Unidos, ya que en este, la oferta de posibles comparables es la más extensa. Los resultados de la búsqueda se presentan en la tabla 2 (ver tabla 2).

Tabla 2. Tasas de instrumentos financieros o de inversión en dólares

\begin{tabular}{|l|c|}
\hline \multicolumn{1}{|c|}{ Instrumento } & $\begin{array}{c}\text { Tasa de interés } \\
\text { promedio anual }\end{array}$ \\
\hline Papel comercial empresas no financieras & $3.00 \%$ \\
\hline Papel comercial empresas financieras & $3.20 \%$ \\
\hline LIBOR 6 meses & $3.66 \%$ \\
\hline Eurodólar 6 meses & $3.80 \%$ \\
\hline Títulos del tesoro 6 meses & $4.10 \%$ \\
\hline Tasa de interés en los mercados de dinero y capitales, con calificación AAA & $5.14 \%$ \\
\hline Tasa Prime & $5.20 \%$ \\
\hline \multicolumn{1}{|c|}{ Rango intercuartil } & \\
\hline Límite inferior & $3.43 \%$ \\
\hline Mediana & $3.80 \%$ \\
\hline Límite superior & $4.62 \%$ \\
\hline
\end{tabular}

Fuente: documentación comprobatoria del contribuyente.

Una vez obtenidas las tasas de interés que se muestran en la tabla 2, el contribuyente señala que utilizó el método de precio comparable no controlado para comparar las tasas de interés pagadas por la subsidiaria en Brasil en las operaciones de préstamo. El contribuyente puntualiza que las tasas de referencia del cuadro representan evidencia de alternativas de inversión en dólares, en donde podrían colocarse los excedentes monetarios de la tesorería del grupo corporativo. Los depósitos, al igual que el papel comercial, también representan alternativas de inversión a los préstamos con su parte relacionada en Brasil. El contribuyente también menciona que ni la casa matriz ni sus subsidiarias en el exterior recibieron financiamiento de entidades no relacionadas, por lo que no existen comparables internos para analizar estas operaciones.

Con base en los resultados mostrados en las tablas 1 y 2 , el contribuyente concluye que el rango intercuartil de las tasas de interés identificadas como comparables 
tiene como límite inferior 3.43\% y como límite superior $4.62 \% .^{3}$ Las tasas devengadas en las operaciones de préstamo entre el contribuyente y su parte relacionada en Brasil durante el año fiscal 2020 van de $4.20 \%$ a $4.52 \%$, y se encuentran dentro del rango anterior. Esto indica que los montos de contraprestación en las operaciones de financiamiento con partes relacionadas son consistentes con los que se hubieran devengado en operaciones comparables entre partes independientes en perfecta sintonía con lo requerido en el artículo 76 IX de la LISR (2013/2020).

\section{Análisis de resultados 4}

Ahora supongamos que la autoridad fiscal en México realiza una auditoría en materia de precios de transferencia con base en la información presentada por el contribuyente en el apartado anterior (en adelante la documentación comprobatoria). De manera inmediata, la autoridad fiscal podría formular el siguiente comentario:

El contribuyente no demuestra haber dado cumplimiento a lo dispuesto por los artículos 76, primer párrafo, fracciones IX y XII; 179, tercero, cuarto y último párrafos, y 180, primer párrafo, fracción I de la LISR vigente en 2020, en sus operaciones de ingresos por intereses devengados a favor con sus partes relacionadas residentes en el extranjero, ni tampoco a lo dispuesto por el artículo 76, primer párrafo, fracción XII, aplicando la metodología establecida en el artículo 180, primer párrafo, fracción I, y considerando para estos efectos lo dispuesto en el artículo 179, tercero y cuarto párrafos conforme a lo establecido por el artículo 180, primer párrafo de la LISR vigente en 2020 en sus operaciones de ingresos por intereses devengados a favor con sus partes relacionadas en el exterior, ya que no demostró que dichas operaciones se hubieran pactado como lo harían con o entre partes independientes en operaciones comparables.

El fundamento de la observación anterior descansa en lo que a continuación se explica.

\footnotetext{
3 El rango intercuartil calculado conforme a lo establecido en el artículo 302 del Reglamento de la Ley del Impuesto sobre la Renta vigente en 2020.

4 Esta sección se presenta en forma de simulación, en la cual la autoridad fiscal en México realiza observaciones a la evidencia preparada por el contribuyente en el apartado anterior. Las posiciones de la autoridad fiscal en este ejercicio de simulación son hipotéticas y no representan la posición de la autoridad fiscal competente en México.
} 


\section{Con respecto a la comparabilidad}

El contribuyente se enfocó en presentar tasas de referencia como rendimientos en inversiones que podrían ser alternativas a canalizar sus excedentes a préstamos con su parte relacionada en Brasil. Su enfoque no fue el de buscar tasas comparables, que es lo que se requiere, sino a presentar el costo de oportunidad de los préstamos otorgados a la subsidiaria en el extranjero. El objetivo es buscar comparables y no un costo de oportunidad. El contribuyente no presentó evidencia de operaciones de préstamo comparables en las que se hubieran pactado dichas tasas de referencia.

El artículo 179, primer párrafo de la LISR (2013/2020) vigente en 2020, señala que:

los contribuyentes que celebren operaciones con partes relacionadas están obligados a determinar sus ingresos acumulables y deducciones autorizadas, considerando para esas operaciones los precios y montos de contraprestaciones que hubieran utilizado con o entre partes independientes en operaciones comparables.

Para dar cumplimiento a este artículo, el contribuyente debió evidenciar que las tasas de referencia presentadas en la documentación comprobatoria hubieran sido utilizadas para pactar un contrato de préstamo entre partes independientes. Debido a esta omisión, se puede determinar un incumplimiento a lo establecido en el primer párrafo del artículo 179 de la LISR (2013/2020).

Adicionalmente, aunque la autoridad fiscal aceptara sin conceder que las tasas de interés presentadas hubiesen sido pactadas en alguna operación de préstamo, no habría forma de determinar las características de las operaciones reales, de tal modo que pudieran aceptarse como comparables. Dentro del elemento de «características de las operaciones» descrito en la fracción I, a) del artículo 179 de la LISR (2013/2020), se debieron analizar elementos tales como 1) monto del principal, 2) plazo, 3) garantías, 4) solvencia del deudor y 5) tasa de interés e, inclusive, las condiciones económicas existentes cuando la operación a la que se hace referencia fue pactada. La evidencia no presenta una comparación que tome en cuenta todas estas características. Adicionalmente, el contribuyente no hizo esfuerzo alguno para realizar los ajustes correspondientes a las tasas de referencia para lograr una mejor comparabilidad entre las operaciones. Debido a esta omisión, se puede determinar un incumplimiento establecido en el tercer párrafo, fracción I, a) del artículo 179 de la LISR (2013/2020). 


\section{Con respecto al margen o spread}

Otra de las fallas importantes en la documentación comprobatoria es la ausencia de la descripción de un margen que debe ser adicionado a la tasa de referencia para determinar el costo integral del financiamiento. Si bien en los contratos de préstamo pactados entre el contribuyente y la parte relacionada en el exterior se hace explicito que la mayoría de los créditos se pactaron a la tasa LIBOR, como tasa de referencia libre de riesgo, una entidad financiera independiente siempre consideraría la adición de un margen para cubrir los costos y riesgos asociados con un préstamo a una empresa domiciliada en el exterior. Como mínimo, el Banco Mundial sostiene que el margen debe incluir una compensación por cada uno de los siguientes apartados (IBRD, 2019):

1. Un margen de ganancia sobre el costo de fondeo.

2. Un margen para cubrir los gastos de apertura del crédito y su posterior administración.

3. Un margen asociado con el plazo del crédito. Este margen estaría directamente asociado con la situación económica/política del país en donde radica el deudor y que pudiera afectar la capacidad y voluntad de pago. Este margen está asociado con el riesgo de crédito del deudor y con el riesgo país.

4. Un margen que cubra el riesgo de un alza en el costo de fondeo cuando este se pacta a un plazo menor al plazo del préstamo.

Como se observa, tres de los cuatro componentes del margen dependen de «aspectos operativos» del préstamo, así como un componente «no operativo» que es el riesgo de crédito del deudor. Con respecto a este punto, esta autoridad fiscal considera que un indicador que aproxima y refleja, de alguna manera, el riesgo de crédito de Brasil es el riesgo país, ya que ante la ausencia de información del riesgo de crédito del deudor, este indicador sería utilizado por una entidad independiente para ajustar una tasa de interés de mercado que pudiera pactar con otra parte independiente (OCDE, 2020). 5

\footnotetext{
${ }^{5}$ La OCDE reconoce que, en la mayoría de los casos, la empresa deudora del préstamo intragrupo no cuenta con una calificación formal emitida por alguna empresa calificadora, por lo que es necesario hacer uso de herramientas e información de dominio público para aproximar el riesgo de crédito. La calificación de riesgo país es de dominio público.
} 
El riesgo-país puede definirse como la amplia gama de riesgos económicos, institucionales, de mercados financieros y legales que se presentan al hacer negocios con o en un país específico y que pueden afectar la calidad crediticia de una entidad no soberana (Standard \& Poors, 2013).

El riesgo-país incorpora el riesgo de impago de la deuda externa soberana (riesgo soberano) y de la deuda externa privada cuando el riesgo de crédito se debe a circunstancias ajenas a la situación de solvencia o liquidez del deudor privado. El riesgo de impago por insolvencia del deudor privado se denomina riesgo comercial o riesgo de insolvencia del cliente. El riesgo país es la calificación de mayor importancia en términos de contenido de información puesto que incorpora la mayor cantidad de aspectos de carácter estructural así como coyuntural de un determinado país.

Sin embargo, surge el problema de que el riesgo país es una calificación de riesgo mayormente cualitativa, en donde, por ejemplo, la calificadora Standard \& Poors utiliza la metodología de «scoring», que toma en cuenta diferentes elementos económicos, políticos e institucionales para llegar a una calificación de riesgo país. La escala que resulta fluctúa entre 1 y 6 , en donde «1» denota riesgo muy bajo y «6», riesgo muy alto (Standard \& Poors, 2017). Debido a la forma en la que se calcula el riesgo país, no es posible «sumarlo» a la tasa de referencia para obtener la «tasa completa» que debe aplicar el contribuyente en su préstamo con su parte relacionada en Brasil.

Ante esta imposibilidad, la autoridad fiscal sugiere tomar como aproximación al riesgo país al riesgo soberano, que incorpora la mayor parte de la información incluida en el riesgo país. A pesar de que el riesgo soberano también se califica de manera cualitativa, es posible migrar de la calificación de riesgo soberano (cualitativa) a un indicador cuantitativo medido en «puntos base», que se lleva a cabo a través del Emerging Markets Bond Index Global (EMBIG), elaborado por J.P. Morgan (J.P. Morgan Securities, 1999). ${ }^{6}$

La justificación de consistencia teórica entre el concepto de riesgo cualitativo y un concepto de riesgo cuantitativo (numérico) se motiva en la evidencia presentada por Norbert Gaillard (2012). Su análisis busca establecer una consistencia entre el EMBIG, calculado por J.P. Morgan con base en los rendimientos de mercado de

\footnotetext{
6 Standard \& Poors, Moody's y Fitch utilizan una combinación de letras y símbolos para denotar la calificación del riesgo soberano como pueden ser AAA, AA+, BBB+, BBB-, C y D, esta última solamente para el caso de Standard \& Poors (Standard \& Poor's, 2020).
} 
los diferentes instrumentos denominados en dólares, incluyendo los Bonos Brady, Eurobonos, Préstamos y emisiones en dólares de mercados locales y el riesgo soberano calculado por las agencias Standard \& Poors, Moody's y Fitch ratings. El análisis de Gaillard incluye una muestra de 32 países, entre ellos México y Brasil, en un período que abarca de diciembre de 1993 a febrero de 2007 (159 meses). Las calificaciones otorgadas por las calificadoras corresponden al final de cada mes, de diciembre de 1993 a febrero de 1997.

La correlación entre los spreads EMBIG que representa la «calificación que otorga el mercado" y los ratings que otorgan las calificadoras se determina a través de un modelo de regresión simple, utilizando datos longitudinales y aplicando el método de mínimos cuadrados ordinarios. El modelo es el siguiente:

$$
\log (E M B I G)_{i t}=a_{i}+\beta R T_{i t}+\varepsilon_{i t}
$$

La variable dependiente $\log (\mathrm{EMBIG})_{\text {it }}$ es el spread, cuya fuente es el EMBIG para los 32 países, y la variable independiente $\log (\mathrm{RAT})_{\text {it }}$ es la calificación de la agencia calificadora para cada uno de los 159 meses considerados en la muestra. Los resultados del modelo se presentan en la tabla 3 (ver tabla 3).

Tabla 3. Resultados del modelo de regresión simple utilizando datos longitudinales y aplicando el método de mínimos cuadrados ordinarios

\begin{tabular}{|l|c|c|c|}
\hline \multicolumn{4}{|c|}{ Log(EMBIG)it $=\alpha+\beta$ RAT $_{\text {it }}+\varepsilon_{\text {it }}$} \\
\hline Calificadora & $\mathbf{( 1 )}$ & $\mathbf{( 2 )}$ & (3) \\
\hline Fitch ( $\beta$ ) & -0.229 & $/$ & $/$ \\
\hline Moody's ( $\beta$ ) & $(61.778)$ & & $/$ \\
& $/$ & -0.231 & $(65.910)$ \\
\hline Standard \& Poors ( $\beta$ ) & $/$ & $/$ & -0.233 \\
& & & $(68.424)$ \\
\hline Constante (a) & 8.471 & 7.767 & 8.297 \\
& $(179.755)$ & $(229.476)$ & $(210.685)$ \\
\hline R cuadrada ajustada & 0.596 & 0.582 & 0.600 \\
\hline \# Observaciones & 2585 & 3124 & 3123 \\
\hline
\end{tabular}

Pruebas «t» en paréntesis debajo del coeficiente. Todos los resultados son significativos con un nivel de confianza del 95\%.

Fuente: Gaillard. (2012). Op. cit., p. 54. 
Los resultados indican que existe una correlación negativa muy robusta entre los spreads del EMBIG y las calificaciones otorgadas por las tres agencias calificadoras combinado con pruebas «t» contundentes. Debido a ello, esta autoridad fiscal utilizará el EMBIG como fuente fundamental de información concerniente al spread asociado con el riesgo país de Brasil en donde se encuentra domiciliada la empresa deudora del contribuyente mexicano (FMl et al., 2017). ${ }^{7}$

\section{Metodología y determinación de precios de transferencia}

Independientemente de las observaciones antes señaladas y con la misma información que el contribuyente preparó para dar cumplimiento a lo establecido en la LISR (2013/2020), la autoridad fiscal hará los supuestos que a continuación se presentan para la determinación de los precios de transferencia (tasa de interés arm's length). ${ }^{8}$

\section{El método de precios de transferencia}

Se aplicará el método de precio comparable no controlado (CUP) establecido en el artículo 180, primer párrafo, fracción I de la LISR (2013/2020) vigente en 2020, tal como lo hizo el contribuyente.

\footnotetext{
7 El riesgo país no es solo un constructo de precios de transferencia, sino una variable real que las empresas tienen en cuenta al realizar inversiones u operaciones con terceros. En el documento se propone un ajuste a los estados financieros de la empresa comparable para incorporar el riesgo país como un ajuste al ROA, siendo este ajuste el diferencial en el rendimiento de los bonos emitidos a diez años, que se consideran libres de riesgo entre el país en donde radica la empresa bajo examen y las comparables. Este diferencial es sumado o restado al ROA para posteriormente proceder al cálculo del rango intercuartil y finalmente al ejercicio de comparabilidad. Estos ajustes solamente se aplican cuando se utilizan los métodos de margen neto operacional, costo incrementado y precio de reventa, y no se aplican al método de precio comparable no vinculado que es el utilizado en el caso bajo revisión en este artículo.

8 Si hubiese una operación comparable previamente identificada, la autoridad fiscal procedería con el análisis de comparabilidad conforme a las etapas descritas en las Directrices de Precios de Transferencia para primeramente determinar los términos y condiciones que dieron origen a la operación controlada. Posteriormente, se compararían estos términos y condiciones con los que existirían si las contrapartes hubieran sido partes no relacionadas realizando una operación comparable. Dado que no existen operaciones comparables para realizar esta comparación, se procede directamente a la tercera etapa que corresponde al cálculo de la tasa de interés arm's length.
} 


\section{Tasas de referencia comparables}

Como se mencionó anteriormente, el contribuyente no demuestra haber considerado operaciones comparables en su análisis de precios de transferencia de los intereses devengados a favor pactados con su parte relacionada en Brasil. Ante la ausencia de operaciones comparables y tomando en cuenta que las tasas de referencia consideradas en la documentación comprobatoria preparada por el contribuyente son las del mercado y, suponiendo sin conceder, que estas tasas se hubieran utilizado en operaciones comparables en las mismas condiciones que el contribuyente, es decir, adicionando un margen, podría inferirse que una entidad independiente podría haber utilizado como parámetro dichas tasas para pactar sus operaciones de financiamiento con partes independientes más un margen. ${ }^{9}$

El contribuyente pactó sus operaciones de financiamiento que le generaron intereses devengados a favor con base únicamente en la tasa LIBOR. La autoridad fiscal considera esta práctica como inaceptable y adopta la posición de que debería agregarse un margen, y que este debería ser similar a uno que partes independientes en operaciones comparables hubieran adicionado a la tasa de mercado de referencia pactada. Este margen se determinaría, entre otros factores, con base en una opinión manifestada por alguna calificadora reconocida. Dado que el contribuyente no proporcionó información financiera o referencia de alguna calificación otorgada por alguna calificadora a su empresa relacionada en Brasil al momento en que se otorgaron financiamientos, esta autoridad fiscal no tiene elementos para determinar el riesgo de crédito.

Por lo anterior, se considera que un indicador que aproxima y refleja de alguna manera el riesgo de crédito, sobre todo para entidades residentes en el extranjero, es el riesgo país, ya que, ante la ausencia de información del riesgo de crédito del

\footnotetext{
${ }^{9}$ La interpretación de la frase «suponiendo sin conceder» implica que la autoridad fiscal hace el supuesto de que la evidencia presentada por el contribuyente cumple con los requisitos establecidos en la LISR (2013/2020). Supone, sin conceder, que las mismas tasas se hubieran utilizado en operaciones comparables bajo las mismas condiciones de préstamo que aquellas efectuadas por el contribuyente. La autoridad fiscal va a utilizar la misma información que presenta el contribuyente y va a darle la misma interpretación al aplicar el método de precios de transferencia, sin que esto implique que ni la información ni su interpretación sean las correctas. Sin embargo, hace la observación de que el argumento de cumplimiento se puede fortalecer al incorporar un margen que correspondería a lo que hubieran pactado partes independientes en operaciones comparables.
} 
deudor, este indicador lo utilizaría una entidad independiente para ajustar una tasa de interés de mercado que pudiera pactar con otra parte independiente. En efecto, cuando un inversionista analiza invertir en un país, la tasa de mercado más el riesgo país, determina la rentabilidad mínima que debe exigir una inversión de tal forma que el inversionista sea indiferente a invertir sus recursos en un país libre de riesgo (Standard \& Poors, 2013, e Iranzo, 2008).

\section{Medición}

La migración de la calificación cualitativa del riesgo soberano a su contraparte cuantitativa (EMBIG) descrita anteriormente tiene una ventaja adicional, además de la estrictamente mecánica, que es la de permitir sumar la prima de riesgo a las tasas de referencia de la misma forma a lo propuesto por el llamado «build-up method» (Pratt y Grabowski, 2014). Esta migración elimina la subjetividad inherente a la calificación soberana determinada por las calificadoras, en especial en el dictamen sobre el riesgo político que enfrenta el país en donde se encuentra la empresa deudora. ${ }^{10}$

Por eso, es preferible utilizar un indicador de mercado no sesgado, como el EMBIG, que se determina por las libres fuerzas del mercado. Es decir, por la interacción de miles de operaciones de oferta y demanda que utilizan toda la información disponible de los diferentes países, que representa el insumo más importante para tomar posiciones. El mercado es el foro en el que se materializan las expectativas de los inversionistas independientes y son millones de decisiones independientes que, en su conjunto, determinan la percepción de este sobre el riesgo país de aquellos que han colocado deuda externa en los mercados internacionales (OCDE, 2020, párr. 10.73 y 10.74). ${ }^{11}$ Por lo anterior, en la tabla 4 se presenta el cálculo de riesgo país promedio para Brasil (ver tabla 4).

10 A partir de este momento, los conceptos riesgo soberano y riesgo país se utilizarán indistintamente.
11 La OCDE reconoce que la metodología utilizada para determinar el riesgo de crédito de fuentes
de dominio público (como el EMBIG) puede diferir significativamente de las metodologías utilizadas
por agencias calificadoras independientes. Por ejemplo, las fuentes públicas de información utilizan
información cuantitativa limitada para determinar el riesgo de crédito por lo que representan opi-
niones de muy corto plazo. Las calificaciones publicadas por agencias calificadoras independientes
se derivan de un análisis mucho más riguroso que considera aspectos estructurales, tanto econó-
micos como políticos e institucionales, por lo que la calificación otorgada es una calificación con
perspectiva de largo plazo. Por eso, la OCDE recomienda que en el proceso para determinar la tasa 
Tabla 4. Determinación del riesgo país para Brasil (puntos base)

\begin{tabular}{|c|c|c|c|c|c|c|c|c|}
\hline $\begin{array}{c}\text { Mes de } \\
\mathbf{2 0 2 0}\end{array}$ & Enero & Febrero & Marzo & Abril & Mayo & Junio & Julio & Promedio \\
\hline $\begin{array}{c}\text { Riesgo } \\
\text { país }\end{array}$ & 217 & 210 & 351 & 421 & 415 & 365 & 366 & 335 \\
\hline
\end{tabular}

Fuente: J.P. Morgan. (2020). Riesgo País EMBI-serie histórica. Los valores representan el promedio de las cotizaciones diarias.

El valor promedio del riesgo país presentado en la tabla 4 puede combinarse con las tasas de referencia de mercado presentadas por el contribuyente en la documentación comprobatoria (ver tabla 2), para llegar a las tasas que partes independientes hubieran pactado en operaciones comparables de financiamiento. Posteriormente, se calculará el rango intercuartil para las mismas para, finalmente, compararlas con las operaciones efectivamente realizadas entre el contribuyente y su parte relacionada en Brasil. Los resultados de lo anterior se muestran en la tabla 5 (ver tabla 5).

Tabla 5. Determinación del rango intercuartil para tasas de interés de mercado, adicionadas con un margen de mercado para el año 2020

\begin{tabular}{|l|c|c|c|}
\hline Instrumento & $\begin{array}{c}\text { Tasa de interés } \\
\text { promedio anual (\%) }\end{array}$ & Riesgo país (\%) & $\begin{array}{c}\text { Tasa de interés promedio } \\
\text { anual ajustada (\%) }\end{array}$ \\
\hline $\begin{array}{l}\text { Papel comercial } \\
\text { empresas no financieras }\end{array}$ & 3.00 & 3.35 & 6.35 \\
\hline $\begin{array}{l}\text { Papel comercial } \\
\text { empresas financieras }\end{array}$ & 3.20 & 3.35 & 6.55 \\
\hline LIBOR 6 meses & 3.66 & 3.35 & 7.01 \\
\hline Eurodólar 6 meses & 3.80 & 3.35 & 7.15 \\
\hline $\begin{array}{l}\text { Títulos del tesoro } \\
\text { 6 meses }\end{array}$ & 4.10 & 3.35 & 7.45 \\
\hline
\end{tabular}

de interés arm's length, se demuestre la consistencia entre ambas metodologías, tanto de fuentes públicas como las que otorgan las calificadoras. 
(continúa)

\begin{tabular}{|c|c|c|c|}
\hline Instrumento & $\begin{array}{c}\text { Tasa de interés } \\
\text { promedio anual (\%) }\end{array}$ & Riesgo país (\%) & $\begin{array}{c}\text { Tasa de interés promedio } \\
\text { anual ajustada (\%) }\end{array}$ \\
\hline $\begin{array}{l}\text { Tasa de interés en los } \\
\text { mercados de dinero } \\
\text { y capitales, con } \\
\text { calificación AAA }\end{array}$ & 5.14 & 3.35 & 8.49 \\
\hline Tasa Prime & 5.20 & 3.35 & 8.55 \\
\hline \multicolumn{4}{|c|}{ Rango intercuartil } \\
\hline Límite inferior & & & 6.78 \\
\hline Mediana & & & 7.15 \\
\hline Límite superior & & & 7.97 \\
\hline
\end{tabular}

Fuente: tablas 1 y 4 . Rango intercuartil calculado con base a la metodología establecida en el artículo 302 del Reglamento de la LISR (2013/2020) vigente en 2020.

De acuerdo con la tabla 5, el rango intercuartil al que partes independientes en operaciones comparables hubieran pactado operaciones de préstamo se encuentra entre 6.78 y $7.97 \%$ con una mediana de $7.15 \%$. Al realizar la comparación con las tasas efectivamente pactadas por el contribuyente con su parte relacionada en Brasil (ver tabla 1), se observa que las tasas efectivamente pactadas en las 12 operaciones reportadas se encuentran fuera de dicho rango, debido a que fluctúan entre el 4.20 y el $4.52 \%$. Por ende, se procede a realizar el ajuste correspondiente, para el cual se considera que la tasa de interés que hubieran utilizado partes independientes en operaciones comparables es la mediana del rango intercuartil a que hace referencia el artículo 276, primer párrafo del Reglamento de la LISR (2013/2020) vigente en 2020, como se puede ver en la tabla 6 (ver tabla 6). 


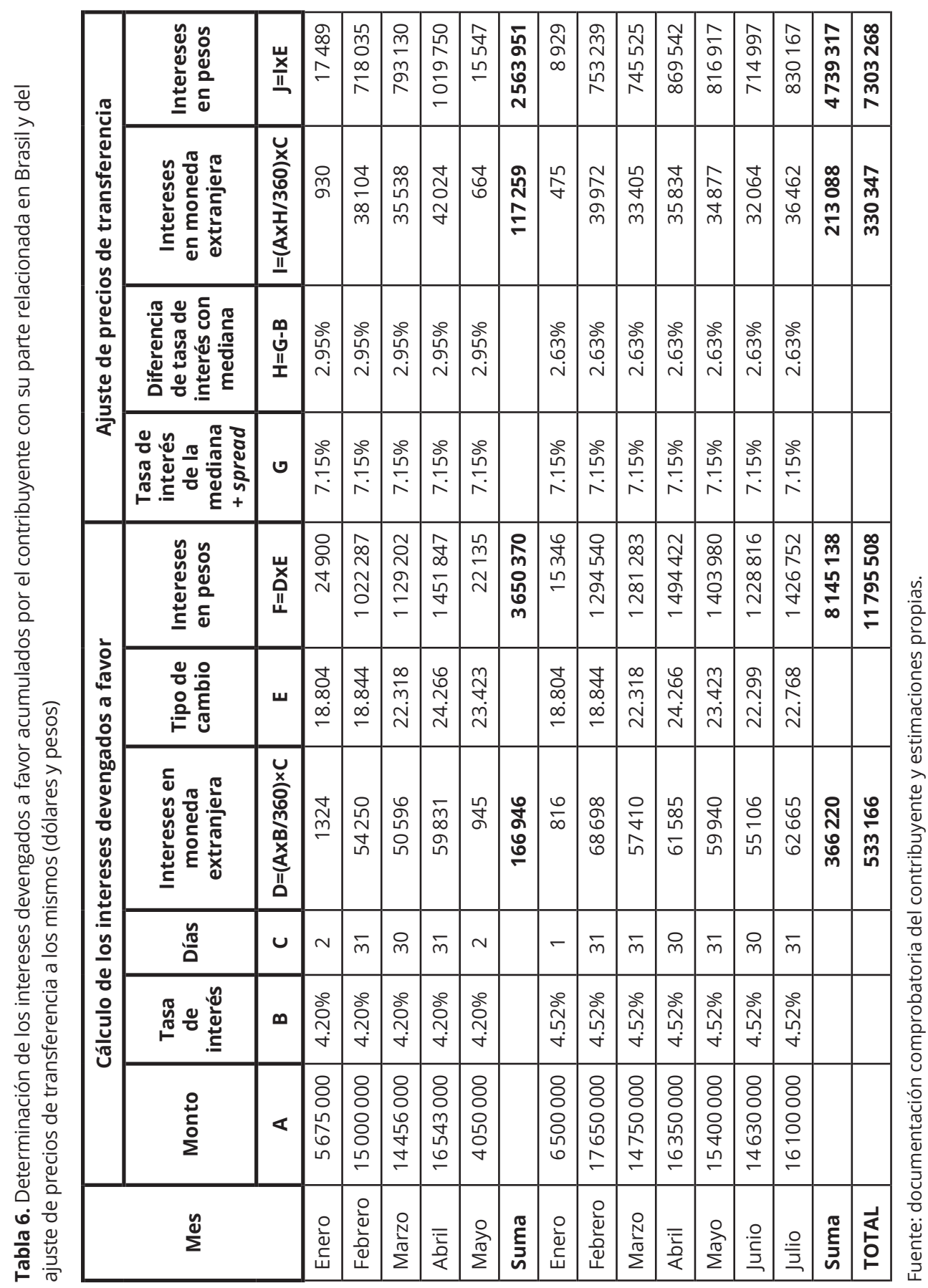




\section{Resumen y conclusiones}

Conforme a las facultades que le otorga el artículo 179 de la LISR (2013/2020) a la autoridad fiscal para determinar los ingresos acumulables y deducciones autorizadas de los contribuyentes, mediante la determinación del precio o monto de la contraprestación en operaciones celebradas entre partes relacionadas, considerando para esas operaciones los precios y montos de las contraprestaciones que hubieran utilizado partes independientes en operaciones comparables, la autoridad fiscal establece que el contribuyente debió haber acumulado el monto por concepto de intereses devengados a favor omitidos como ingresos acumulables por un monto de MX 7303 268.00. Este resultado inmediatamente se transforma en un crédito fiscal para el contribuyente con las obligaciones legales establecidas en la normatividad fiscal vigente. ${ }^{12}$

\section{Comentarios finales}

Ante la falta de un contrato de préstamo pactado entre partes independientes que pudiera ser comparable, se procedió a la construcción de una tasa de interés arm's length a partir de la información presentada por el contribuyente, combinada con las recomendaciones propuestas en el reporte Transfer Pricing Guidance on Financial Transactions (OCDE, 2020). Son tres las recomendaciones a las que se recurrió en la presentación del caso:

1. La primera es considerar que el producto subyacente de las tasas de interés de referencia, presentadas por el contribuyente en la evidencia comprobatoria, podrían considerarse como préstamos entre partes independientes.

2. En ausencia de una calificación crediticia de la empresa deudora por parte de una empresa calificadora, se aproxima el riesgo de crédito a través del riesgo país utilizando información de dominio público. Sobre este punto y conforme a la recomendación planteada por la OCDE (2020) en el párrafo núm. 10.74 del citado documento, se presenta evidencia de la consistencia entre la información

\footnotetext{
12 La conclusión del presente caso no debe generalizarse a otras situaciones de la misma naturaleza ya que descansa íntegramente en los supuestos sobre la calidad de la información presentada por el contribuyente. Cualquier diferencia en la misma, podría dar lugar a conclusiones diferentes.
} 
pública utilizada y le metodología de calificación cualitativa presentada por las empresas calificadoras más importantes del mercado.

3. La construcción de una tasa de interés «completa», que incluye la tasa de referencia más el margen de forma similar a lo propuesto por el llamado «build up method». Posteriormente, se procede a realizar el cálculo del rango intercuartil para finalmente aplicar el ajuste por precios de transferencia a partir de la mediana de las tasas comparables que representa la tasa de interés arm's length.

De esta forma, este artículo presentó una opción, resultado de un balance entre lo establecido en la LISR (2013/2020) y las nuevas Directrices de la OCDE sobre transacciones financieras, que le permita al contribuyente y a la autoridad fiscal fortalecer las posiciones propias en procesos de auditoría. En el caso expuesto en este artículo, la alternativa de «construcción de comparables» se presenta de manera aleatoria por la autoridad fiscal y no por el contribuyente. No obstante, la alternativa bien puede presentarse desde el punto de vista del contribuyente. 


\section{Referencias bibliográficas}

Durst, M. (2012). Speaking of Problems with the OECD Transfer Pricing Guidelines (Sobre los problemas con las Directrices de transferencias de precios de la OCDE). [Video]. Vimeo. https://vimeo.com/45954220

Eden, L., Bahar, G. y Bahar, A. (2019). The Economics of Transfer Pricing (La economía de la transferencia de precios). Edward Elgar Publishing, Ltd. https://www. e-elgar.com/shop/gbp/the-economics-of-transfer-pricing-9781840648324.html

Fondo Monetario Internacional (FMI), Grupo Banco Mundial (GBM), Organización para la Cooperación y el Desarrollo Económico (OCDE) y Organización de las Naciones Unidas (ONU). (2017). Plataforma de colaboración en materia tributaria: Guía práctica para afrontar las dificultades asociadas con la falta de comparables en los análisis de precios de transferencia. https://www.oecd.org/tax/guia-practicacomparables-precios-de-los-minerales.pdf

Gaillard, N. (2012). A Century of Sovereign Ratings (Un siglo de calificaciones soberanas). Springer Science + Business Media LLC. https://www.springer.com/gp/ book/9781461405221

International Bank for Reconstruction and Development (IBRD). (2016). Transfer Pricing and Development Economies. A Handbook for Policy Makers and Practitioners. https://openknowledge.worldbank.org/handle/10986/25095?locale-attribute=es

International Bank for Reconstruction and Development (IBRD). (2019). Memorandum from the President. Subject: IBRD Lending Rates and Spreads Applicable on or After April 1 (Memorándum del presidente. Tema: tasas de préstamos y spreads aplicables del IBRD en o después del 1 de abril). http://documents1.worldbank. org/curated/en/842221556637823217/pdf/IBRD-Lending-Rates-and-SpreadsApplicable-on-or-after-April-1-2019.pdf

Iranzo, S. (2008). Introducción al riesgo país. Documento ocasional No. 0802. Banco de España. https://www.bde.es/f/webbde/SES/Secciones/Publicaciones/PublicacionesSeriadas/DocumentosOcasionales/08/Fic/do0802.pdf

J.P. Morgan Securities Inc. (1999). Introducing the J.P. Morgan Emerging Markets Bond Index Global (EMBI Global). (Introducción del índice global de bonos de mercados emergentes de J.P. Morgan) [Informe metodológico] https://faculty.darden. virginia.edu/liw/emf/embi.pdf

J.P. Morgan Securities Inc. (2020). Riesgo país EMBI-Serie histórica. https://www. invenomica.com.ar/riesgo-pais-embi-america-latina-serie-historica/ 
Ley del Impuesto Sobre la Renta. (LISR). 2013, última reforma, 2020. http://www. diputados.gob.mx/LeyesBiblio/pdf/LISR_081220.pdf

Naciones Unidas. (2013). Practical Manual on Transfer Pricing for Developing Countries (Manual práctico sobre la transferencia de precios para países en desarrollo). https://www.un.org/esa/ffd/wp-content/uploads/2017/04/Manual-TP-2017.pdf

Organización para la Cooperación y el Desarrollo Económicos (OCDE). (2017a). Modelo de Convenio Tributario sobre la Renta y sobre el Patrimonio: Versión abreviada. OCDE/Instituto de Estudios Fiscales. https://www.oecd-ilibrary.org/taxation/ modelo-de-convenio-tributario-sobre-la-renta-y-sobre-el-patrimonio-versionabreviada-2017_765324dd-es

Organización para la Cooperación y el Desarrollo Económicos (OCDE). (2017b). Directrices de la OCDE aplicables en materia de precios de transferencia a empresas multinacionales y administraciones tributarias 2017. OCDE/Instituto de Estudios Fiscales. https://www.oecd-ilibrary.org/taxation/directrices-de-la-ocde-aplicables-en-materiade-precios-de-transferencia-a-empresas-multinacionales-y-administracionestributarias-2017_9788480083980-es

Organización para la Cooperación y el Desarrollo Económicos (OCDE). (2020). Transfer Pricing Guidance on Financial Transactions: Inclusive Framework on BEPS: Actions 4, 8-10. (Directrices de transferencia de precios en transacciones financieras; marco inclusivo en BEPS; acciones 4, 8-10) http://www.oecd.org/tax/ beps/transfer-pricing-guidance-on-financial-transactions-inclusive-framework-onbeps-actions-4-8-10.htm

Padhi, S.K. (2019). «Transfer Pricing a Review of the Literature» (Transferencia de precios, revisión de la literatura). International Journal of Advanced Research in Management, 10 (1), 1-7. https://www.iaeme.com/MasterAdmin/uploadfolder/ IJARM_10_10_001/JARM_10_10_001.pdf

Pratt, P. S. y Grabowski, R. (2014). Cost of Capital: Applications and Examples + Website (Costo del capital: aplicaciones y ejemplos + sitio web). (5. a ed.). Cap. 9. https:// www.oreilly.com/library/view/cost-of-capital/9781118555804/23_chapter-09. html\#ch009-sec001

Silva, E. (2019). «Location Savings Adjustment to Profits» (Ajuste de ahorros por ubicación a las ganancias), Journal of International Business and Economics, vol. 19. https://www.researchgate.net/publication/331455044_LOCATION_SAVINGS_ ADJUSTMENT_TO_PROFITS

Standard \& Poors (S\&P). (2013). Country Risk Assessment. Methodology and Assumptions (Evaluación de riesgo país. Metodología y supuestos). S\&P Rating 
Services. McGraw Hill Financial. https://www.maalot.co.il/publications/MT201 31127143757.pdf

Standard \& Poors (S\&P). (2017). Sovereign Rating Methodology (Metodología de calificación soberana). S\&P Global Ratings. https://enterprise.press/wp-content/ uploads/2017/05/Sovereign-Rating-Methodology.pdf

Standard \& Poors (S\&P). (2020). Definición de calificaciones. S\&P Global Ratings. https://www.standardandpoors.com/es_LA/delegate/getPDF?articleld=2318 977\&type=COMMENTS\&subType=RATING\%20DEFINITION\#: :text=Una\%20 calificaci\%C3\%B3n\%20crediticia\%20de\%20emis 


\section{Sobre el autor}

Egresado de la licenciatura de economía de la Universidad Anáhuac. Maestría y doctorado en la misma especialidad por la Universidad de Georgetown, en Washington, D.C. Se desempeñó como economista en el Banco Interamericano de Desarrollo en Washington, D.C. y en la oficina de representación del Banco Mundial en México. Fue director general de la Organización para la Cooperación y el Desarrollo Económicos (OCDE) y para los proyectos del Plan Puebla Panamá en la Secretaría de Relaciones Exteriores. Fue fiscalizador de precios de transferencia en el Servicio de Administración Tributaria. Su último encargo público fue la vicepresidencia de Administración y Planeación en la Comisión Nacional Bancaria y de Valores. Actualmente es profesor e investigador de la Facultad de Economía y Negocios de la Universidad Anáhuac-México. Tiene en su haber diversas publicaciones en revistas y capítulos en libros.

luis.landaf@anahuac.mx

https://orcid.org/0000-0002-0932-7734 